\title{
Generalized inverse Lindley power series distributions: modeling and simulation
}

\author{
Said H. Alkarni \\ Department of Quantitative Analysis, King Saud University, Riyadh, Saudi Arabia.
}

\begin{abstract}
In this paper, we introduce a new generalization of a class of inverse Lindley distributions called the generalized inverse Lindley power series (GILPS) distribution. This class of distributions is obtained by compounding the generalized class of inverse Lindley distributions with the power series family of distributions. The GILPS contains several lifetime subclasses such as inverse Lindley power series, two parameters inverse Lindley power series, and inverse power Lindley power series distributions. It can generate many statistical distributions such as the inverse power Lindley Poisson distribution, the inverse power Lindley geometric distribution, the inverse power Lindley logarithmic distribution, and the inverse power Lindley binomial distribution. The proposed class has flexibility in the sense that it can generate new lifetime distributions as well as some existing distributions. For the proposed class, several properties are derived such as hazard rate function, limiting behavior, quantile function, moments, moments generating function, and distributions of order statistics. The method of maximum likelihood estimation can be used to estimate the model parameters of this new class. A simulation for a selective model will be discussed. At the end, we will demonstrate applications of three real data sets to show the flexibility and potential of the new class of distributions.
\end{abstract}

Keywords: Generalized inverse Lindley power series distributions, inverse Lindley power series distributions, inverse power Lindley power series distribution.

2010 MSC: 62E15, 60E05.

(C)2019 All rights reserved.

\section{Introduction}

Consider the lifetime, $X_{i}$, of a system with $N$ components. The life of each component is a positive continuous random variable and can have any lifetime distribution. Examples might include the exponential, the gamma, the Weibull, the Lindley, the inverse Weibull, the inverse gamma, and the inverse Lindley. The life of such a system can be modeled as a non-negative random variable $X=\min \left\{X_{i}\right\}_{i=1}^{N}$ or $Y=\max \left\{X_{i}\right\}_{i=1}^{N}$ based on whether the components are in a series or parallel. The distribution of the discrete random variable $\mathrm{N}$ can be any truncated discrete distribution such as the zero-truncated Poisson, the geometric, the logarithmic, the binomial and the generalized power series. The continuous random variables $X_{i}, i=1, \ldots, N$ are independent from the random variable $N$.

The inverted family of distributions is commonly used to model data from reliability experiments that exhibit unimodal hazard rate functions. Lindley [21] suggested a new distribution as an alternative for

Email address: salkarni@ksu.edu.sa (Said H. Alkarni)

doi: $10.22436 /$ jnsa.012.12.03

Received: 2018-06-30 Revised: 2018-10-05 Accepted: 2018-10-08 
the exponential distribution that became the well-known Lindley distribution. Since then, many other generalizations and extensions of the Lindley distributions have been proposed, both to improve its flexibility and to achieve a better fitting of lifetime data. The inverse Lindley (IL) distribution was originally proposed by Sharma et al. [29]. Sharma et al. [30] added a shape parameter to the IL distribution, and this work was extended by Alkarni [1]. Another extension of the inverse Lindley distribution was proposed by Sharma and Khandelwal [28]. Recently, Barco et al. [5] introduced the inverse power Lindley (IPL) distribution and applied it to real data. It showed good flexibility in fitting the data compared with many existing distributions.

In recent years, several power series distributions have been proposed in the literature. These distributions were obtained by compounding some useful lifetime distributions with power series distributions. Lindley power series (LPS) class of distributions (Liyanage and Pararai [22]), Weibull power series class of distributions (Morais and Barreto-Souza [25]), compound class of extended Weibull power series of distributions (Silva et al. [31]), a generalization of the extended Weibull power series family of distributions (Alkarni [2]), exponentiated extended Weibull power series class of distributions (Tahmasebi and Jafari [33]), inverse Weibull power series distributions (Shafie et al. [27]), generalized exponential power series of distributions (Mahmoudi and Jafari [23]), complementary exponential power series (Flores et al. [14]), double-bounded Kumaraswamy power series (Bidram and Nekoukhou [6]), Burr XII power series (Silva and Cordeiro [32]), generalized linear failure rate power series of distributions (Alamatsaz and Shams [16]), Birnbaum Saunders power series of distribution (Bourguignon et al. [7]), linear failure rate-power series of distributions (Mahmoudi and Jafari [24]), complementary extended Weibull-power series of distributions (Cordeiro and Silva [8]), Gompertz-power series distributions (Tahmasebi and Jafari [19]), the Exponential Pareto power series distribution (Elbatal et al. [13]), generalized modified Weibull power series distribution (Bagheri et al. [4]), Compound family of generalized inverse Weibull power series distributions (Hassan et al. [18]), and complementary exponentiated inverted Weibull power series family of distributions (Hassan et al. [17]) are some examples of such distributions. To compound a continuous distribution with a discrete one, Nadarajah et al. [26] introduced the package: Compounding in R software (R Development Core Team [34]).

In this paper, we introduce the GILPS by considering a system with parallel components and by compounding the generalized class of inverse Lindley distributions with the power series distributions. The GILPS class of distributions is a flexible family and contains several inverse Lindley types of distributions compounded with discrete distributions (truncated at zero).

The proposed family of distributions can be applied to many fields such as business, environmental science, actuarial science, biomedical studies, demography and industrial reliability. This family contains several subclasses and lifetime models as special cases. In addition, it gives us the flexibility to choose any compound lifetime for modeling many behavioral types of lifetime data.

The remainder of this paper is organized as follows. In Section 2, we define the generalized inverse Lindley (GIL) class of distributions and present some existing models that can be deduced as special cases of the considered model. In Section 3, we define the GILPS class of distributions in terms of the cumulative distribution function and introduce some special subclasses. In Section 4, we provide the general properties of the GILPS class, including density, hazard function, quantile function, moments, moments generating function and distribution of order statistics. The estimations of GILPS parameters are investigated in Section 5 using the method of maximum likelihood estimation. In Section 6, some special distributions are introduced as examples of the GILPS. In Section 7, a simulation is applied to a GILPS model to test the performance of the maximum likelihood method in estimating the parameters. In Section 8, we present some real data to illustrate the applicability and flexibility of the GILPS distributions. Finally, some concluding remarks are offered in Section 9. 


\section{The generalized inverse Lindley class of distributions}

In this section, we define the generalized inverse Lindley (GIL) class of distributions, which generates most of the existing inverse Lindley types of distributions and can be used to generate new ones.

Definition 2.1. The GIL class of distributions is defined by its cumulative distribution function (cdf) as follows:

$$
G(x ; \beta, \lambda, \alpha)=\frac{\beta+\lambda+\beta \lambda x^{-\alpha}}{\beta+\lambda} e^{-\beta x^{-\alpha}}, \quad \beta, \lambda, \alpha, x>0 .
$$

The corresponding probability distribution function (pdf) becomes:

$$
g(x ; \beta, \lambda, \alpha)=\frac{\alpha \beta^{2}}{\beta+\lambda}\left(x^{-\alpha-1}+\lambda x^{-2 \alpha-1}\right) e^{-\beta x^{-\alpha}}, \quad \beta, \lambda, \alpha, x>0 .
$$

We note that the GIL class of distributions was introduced as a three-parameter model called "the extended inverse Lindley distribution" by Alkarni [1].

Several inverse Lindley distributions can be written in form (2.1) depending on the parameters $\lambda$ and $\alpha$. We present some special distributions in the following sub-section.

\subsection{Special cases}

\subsubsection{Inverse Lindley distribution}

For the choice of $\lambda=\alpha=1$, the cdf in (2.1) becomes:

$$
F(x ; \beta)=\left(\frac{\beta+1+x^{-1}}{\beta+1}\right) e^{-\beta x^{-1}}, \quad \beta, x>0,
$$

which is the cdf of the inverse Lindley distribution introduced by Sharma et al. [29]. From (2.2), the pdf is given by

$$
f(x ; \beta)=\frac{\beta^{2}}{\beta+1}\left(x^{-2}+x^{-3}\right) e^{-\frac{\beta}{x}}, \beta, x>0 .
$$

\subsubsection{Two-parameter inverse Lindley distribution}

For the choice of $\alpha=1$, the cdf in (2.1) becomes:

$$
F(x ; \beta, \lambda)=\frac{\beta+\lambda+\beta \lambda x^{-1}}{\beta+\lambda} e^{-\beta x^{-1}}, \beta, \lambda, x>0,
$$

which is the cdf of the two parameters of the inverse Lindley. From (2.2), the pdf is given by:

$$
f(x ; \beta, \lambda)=\frac{\beta^{2}}{\beta+\lambda}\left(x^{-2}+\lambda x^{-3}\right) e^{-\beta x^{-1}}, \beta, \lambda, x>0 .
$$

\subsubsection{Inverse power Lindley distribution}

If $\lambda=1$ then the cdf in (2.1) becomes:

$$
F(x ; \beta, \alpha)=\left[\frac{\beta+1+\beta x^{-\alpha}}{\beta+1}\right] e^{-\beta x^{-\alpha}}, \quad \beta, \alpha, x>0,
$$

which is the cdf of the inverse power Lindley distribution introduced by Barco et al. [5]. From (2.2), the pdf is given by:

$$
f(x ; \beta, \alpha)=\frac{\alpha \beta^{2}}{\beta+1}\left[x^{-\alpha-1}+x^{-2 \alpha-1}\right] e^{-\beta x^{-\alpha}}, \quad \beta, \alpha, x>0 .
$$




\section{The GILPS family}

In this section, we derive the family of GILPS distributions by compounding the GIL class of distributions with the power series distributions.

Let $\mathrm{N}$ be a zero-truncated discrete random variable having a power series distribution with the following probability mass function:

$$
p_{n}=p(N=n)=\frac{a_{n} \theta^{n}}{c(\theta)}, n=1,2, \ldots
$$

where $a_{n} \geqslant 0$ depends only on $n, c(\theta)=\sum_{n=1}^{\infty} a_{n} \theta^{n}$, and $\theta \in(0, s)$ is chosen in such a way that $c(\theta)$ is finite. The power series family of distributions, includes Poisson, geometric, logarithmic and binomial distributions, see Johnson et al. [20]. Useful quantities, such as $a_{n}, c(\theta)$, the first derivative of $c(\theta)$, and its inverse, for the above mentioned distributions truncated for zero are presented in Table 1.

Table 1: Useful quantities for some power series distributions.

\begin{tabular}{|l|c|c|c|c|c|}
\hline Distribution & $a_{n}$ & $c(\theta)$ & $c^{\prime}(\theta)$ & $c^{-1}(\theta)$ & par. space \\
\hline Poisson & $\frac{1}{n !}$ & $e^{\theta}-1$ & $e^{\theta}$ & $\log (\theta+1)$ & $(0, \infty)$ \\
\hline Geometric & 1 & $\frac{\theta}{1-\theta}$ & $\frac{1}{(1-\theta)^{2}}$ & $\frac{\theta}{(\theta+1)}$ & $(0,1)$ \\
\hline Logarithmic & $\frac{1}{n}$ & $-\log (1-\theta)$ & $\frac{1}{(1-\theta)}$ & $1-e^{-\theta}$ & $(0,1)$ \\
\hline Binomial & $\left(\begin{array}{c}m \\
n\end{array}\right)$ & $(1+\theta)^{m}-1$ & $m(1+\theta)^{m-1}$ & $(\theta+1)^{\frac{1}{m}}-1$ & $\mathrm{~N}^{+}$ \\
\hline
\end{tabular}

Given $N$, let $X=\max \left(X_{1}, \ldots, X_{N}\right)$, where $X_{i}, i=1, \ldots, N$ are independent and identically distributed (iid) random variables with cdf as in (2.1). Then the cdf of $X \mid N=n$ is given by:

$$
F_{X \mid N=n}(x)=[G(x ; \beta, \lambda, \alpha)]^{n}=\left(\frac{\beta+\lambda+\beta \lambda x^{-\alpha}}{\beta+\lambda}\right)^{n} e^{-\beta x^{-\alpha}}, x>0, n \geqslant 1 .
$$

The GILPS distribution is then defined by the marginal cdf of $X$, which is given by:

$$
\begin{aligned}
F(x ; \theta, \beta, \lambda, \alpha) & =\sum_{n=1}^{\infty} \frac{a_{n} \theta^{n}}{c(\theta)}[G(x ; \beta, \lambda, \alpha)]^{n} \\
& =\frac{c(\theta G(x ; \beta,, \lambda, \alpha))}{c(\theta)}=\frac{c\left(\frac{\theta\left(\beta+\lambda+\beta \lambda x^{-\alpha}\right)}{\beta+\lambda} e^{-\beta x^{-\alpha}}\right)}{c(\theta)}, \theta, \beta, \lambda, \alpha, x>0 .
\end{aligned}
$$

Remark 3.1. $\operatorname{Let} X_{(2.1)}=\min \left\{X_{i}\right\}_{i=1}^{N}$. Then, the cdf of $X_{(2.1)}$ is given by:

$$
\begin{aligned}
F_{X_{(1)}}(x ; \theta, \beta,, \lambda, \alpha) & =\sum_{n=1}^{\infty} p_{n}(1-G(x ; \theta, \beta, \lambda, \alpha))^{n} \\
& =1-\frac{c(\theta(1-G(x ; \theta, \beta,, \lambda, \alpha)))}{c(\theta)}=1-\frac{c\left(\theta\left[1-\frac{\beta+\lambda+\beta \lambda x^{-\alpha}}{\beta+\lambda} e^{-\beta x^{-\alpha}}\right]\right)}{c(\theta)}, \quad \theta, \beta, \lambda, \alpha, x>0 .
\end{aligned}
$$

Based on the choice of $c(\theta), \lambda$, and $\alpha$ with form (3.1), this class covers the entire compounded inverse Lindley types of distributions.

Remark 3.2. Setting $\lambda=\alpha=1$, we obtain the inverse Lindley power series (ILPS) class of distributions. Setting $\alpha=1$, we have the two parameters of the inverse Lindley power series (TILPS) class of distributions. Setting $\lambda=1$, we have the inverse power Lindley power series (IPLPS) class of distributions. 


\section{General properties}

\subsection{Density and hazard functions}

The pdf and hazard function (hf) of the GILPS are, respectively, given by:

$$
\begin{aligned}
f(x ; \theta, \beta, \lambda, \alpha) & =\theta g(x ; \theta, \beta, \lambda, \alpha) \frac{c^{\prime}(\theta G(x ; \theta, \beta, \lambda, \alpha))}{c(\theta)} \\
& =\frac{\alpha \theta \beta^{2}}{\beta+\lambda}\left(x^{-\alpha-1}+\lambda x^{-2 \alpha-1}\right) e^{-\beta x^{-\alpha}} \frac{c^{\prime}\left(\frac{\theta\left(\beta+\lambda+\beta \lambda x^{-\alpha}\right)}{\beta+\lambda} e^{-\beta x^{-\alpha}}\right)}{c(\theta)},
\end{aligned}
$$

and

$$
\begin{aligned}
\tau(x ; \theta, \beta,, \lambda, \alpha) & =\frac{f(x ; \theta, \beta,, \lambda, \alpha)}{1-F(x ; \theta, \beta, \lambda, \alpha)} \\
& =\theta g(x ; \theta, \beta, \lambda, \alpha) \frac{c^{\prime}(\theta G(x ; \theta, \beta, \lambda, \alpha))}{c(\theta)-c(\theta G(x ; \theta, \beta, \lambda, \alpha))} \\
& =\frac{\alpha \theta \beta^{2}}{\beta+\lambda}\left(x^{-\alpha-1}+\lambda x^{-2 \alpha-1}\right) e^{-\beta x^{-\alpha}} \frac{c^{\prime}\left(\frac{\theta\left(\beta+\lambda+\beta \lambda x^{-\alpha}\right)}{\beta+\lambda} e^{-\beta x^{-\alpha}}\right)}{c(\theta)-c\left(\frac{\theta\left(\beta+\lambda+\beta \lambda x^{-\alpha}\right)}{\beta+\lambda} e^{-\beta x^{-\alpha}}\right)} .
\end{aligned}
$$

Proposition 4.1. The limiting distribution of the GILPS when ' $\rightarrow 0^{+}$is the GIL.

Proof. Using the L'Hôpital's rule

$$
\begin{aligned}
& \lim _{\rightarrow 0^{+}} F(x ; \theta, \beta, \lambda, \alpha)=\lim _{\rightarrow 0^{+}} \frac{c(\theta G(x ; \theta, \beta, \lambda, \alpha))}{c(\theta)} \\
& =\lim _{\rightarrow 0^{+}} \frac{\sum_{n=1}^{\infty} a_{n} \theta^{n}(G(x ; \theta, \beta, \lambda, \alpha))^{n}}{\sum_{n=1}^{\infty} a_{n} \theta^{n}} \\
& =\lim _{\rightarrow \rightarrow 0^{+}} \frac{a_{1}(G(x ; \theta, \beta, \lambda, \alpha))+\sum_{n=2}^{\infty} n a_{n} \theta^{n-1}(G(x ; \theta, \beta, \lambda, \alpha))^{n}}{a_{1}+\sum_{n=2}^{\infty} n a_{n} \theta^{n-1}} \\
& =G(x ; \theta, \beta, \lambda, \alpha)=\frac{\beta+\lambda+\beta \lambda x^{-\alpha}}{\beta+\lambda} e^{-\beta x^{-\alpha}} \text {, }
\end{aligned}
$$

which is the cdf of the GIL distribution.

Proposition 4.2. The $p d f$ of GILPS distributions can be expressed as an infinite number of linear combinations of densities of the order statistics and given by

$$
\begin{aligned}
f(x ; \theta, \beta, \lambda, \alpha)= & \sum_{n=1}^{\infty} \sum_{i=0}^{n-1}\left(\begin{array}{c}
n-1 \\
i
\end{array}\right) \frac{n a_{n} \theta^{n}}{c(\theta)}(\beta+\lambda)^{i-n} \alpha \beta^{2}(\beta \lambda)^{n-i-1} \\
& \times\left(x^{-n \alpha+i \alpha-1}+\lambda x^{-n \alpha+i \alpha-\alpha-1}\right) e^{-n \beta x^{-\alpha}}, \quad \theta, \beta, \lambda, \alpha>0 .
\end{aligned}
$$

Proof. Given that $c^{\prime}(\theta)=\sum_{n=1}^{\infty} n a_{n} \theta^{n-1}$, we have,

$$
f(x)=\theta g(x ; \theta, \beta, \lambda, \alpha) \frac{c^{\prime}(\theta G(x ; \theta, \beta,, \lambda, \alpha))}{c(\theta)}=\sum_{n=1}^{\infty} p(N=n) g_{Y}(x ; n),
$$

where $g_{Y}(x ; n)$ is the pdf of $Y=\max \left(Y_{1}, \ldots, Y_{n}\right)$, and

$$
g_{Y}(x ; n)=n g(x ; \beta, \lambda, \alpha)(G(x ; \beta, \lambda, \alpha,))^{n-1}=\frac{n \alpha \beta^{2}}{\beta+\lambda}\left(x^{-\alpha-1}+\lambda x^{-2 \alpha-1}\right) e^{-\beta x^{-\alpha}}\left[\frac{\beta+\lambda+\beta \lambda x^{-\alpha}}{\beta+\lambda} e^{-\beta x^{-\alpha}}\right]^{n-1} .
$$


Hence

$$
f(x ; \theta, \beta, \lambda, \alpha)=\sum_{n=1}^{\infty} \frac{n a_{n} \theta^{n}}{c(\theta)} \frac{\alpha \beta^{2}}{(\beta+\lambda)^{n}}\left(x^{-\alpha-1}+\lambda x^{-2 \alpha-1}\right) \quad\left[\beta+\lambda+\beta \lambda x^{-\alpha}\right]^{n-1} e^{-n \beta x^{-\alpha}},
$$

and using the binomial expansion, the pdf of the GILPS can be written as

$$
\begin{aligned}
f(x ; \theta, \beta, \lambda, \alpha)= & \sum_{n=1}^{\infty} \sum_{i=0}^{n-1}\left(\begin{array}{c}
n-1 \\
i
\end{array}\right) \frac{n a_{n} \theta^{n}}{c(\theta)}(\beta+\lambda)^{i-n} \alpha \beta^{2}(\beta \lambda)^{n-i-1} \\
& \times\left(x^{-n \alpha+i \alpha-1}+\lambda x^{-n \alpha+i \alpha-\alpha-1}\right) e^{-n \beta x^{-\alpha}}, \quad \theta, \beta, \lambda, \alpha>0 .
\end{aligned}
$$

\subsection{Moments, and moments-generating function}

The $r^{\text {th }}$ moment of a random variable $X \sim \operatorname{GILPS}(\theta, \beta, \lambda, \alpha)$ distribution, $\mu_{r}^{\prime}$, is obtained using (4.3) as:

$$
\begin{aligned}
\mu_{r}^{\prime} & =\int_{0}^{\infty} x^{r} f_{X}(x) d x \\
& =\sum_{n=1}^{\infty} \sum_{i=0}^{n-1}\left(\begin{array}{c}
n-1 \\
i
\end{array}\right) \frac{a_{n} \theta^{n}}{c(\theta)} n^{\frac{r}{\alpha}-n+i} \beta^{\frac{r}{\alpha}} \lambda^{n-i-1}(\beta+\lambda)^{i-n}\left[n \beta+\lambda\left(n-\frac{r}{\alpha}-i\right)\right] \Gamma\left(n-\frac{r}{\alpha}-i\right) .
\end{aligned}
$$

The moments generating function (mgf) of the GILPS is obtained as $M_{X}(t)=E\left(e^{t x}\right), t>0$. Using the series expansion $e^{t x}=\sum_{k=0}^{\infty} \frac{t^{k} x^{k}}{k !}$, the above expressions are reduced to:

$$
M_{X}(t)=\sum_{k=0}^{\infty} \sum_{n=1}^{\infty} \sum_{i=0}^{n-1}\left(\begin{array}{c}
n-1 \\
i
\end{array}\right) \frac{t^{k}}{k !} \frac{a_{n} \theta^{n}}{c(\theta)} n^{\frac{k}{\alpha}-n+i} \beta^{\frac{k}{\alpha}} \lambda^{n-i-1}(\beta+\lambda)^{i-n}\left[n \beta+\lambda\left(n-\frac{k}{\alpha}-i\right)\right] \Gamma\left(n-\frac{k}{\alpha}-i\right),
$$

where $\Gamma \mathrm{a}=\int_{0}^{\infty} x^{\mathrm{a}-1} e^{-x} \mathrm{~d} x$.

Therefore, the mean and the variance of the GILPS distribution, respectively can be obtained easily from (4.4) as $\mu=\mu_{1}{ }^{\prime}$ and $\sigma^{2}=\mu_{2}{ }^{\prime}-\mu^{2}$.

The skewness and kurtosis measures can be obtained from the expressions

$$
\text { skewness }=\frac{\mu_{3}^{\prime}-3 \mu_{2}^{\prime} \mu+2 \mu^{3}}{\sigma^{3}}, \quad \text { curtosis }=\frac{\mu_{4}^{\prime}-4 \mu_{3}^{\prime} \mu+6 \mu_{2}^{\prime} \mu^{2}-3 \mu^{4}}{\sigma^{4}},
$$

upon substituting for the raw moments from (4.4).

\subsection{Quantile function and order statistics}

In this section, the quantile function and order statistics of GILPS distributions will be derived.

Proposition 4.3. Let $\mathrm{X} \sim \mathrm{GILPS}(\theta, \beta, \lambda, \alpha)$ have the cdf as in (3.1). The quantile function of $\mathrm{X}$ is given by:

$$
\mathrm{Q}_{x}(\mathrm{p})=\left[-\frac{1}{\lambda}-\frac{1}{\beta}-\frac{1}{\lambda \beta} W_{-1}\left(\frac{-(\beta+\lambda) \mathrm{c}^{-1}(\mathrm{pc}(\theta))}{\theta \mathrm{e}^{\beta+\lambda}}\right)\right]^{-\frac{1}{\alpha}}
$$

where $W_{-1}($.$) is the negative branch of the Lambert W$ function (see Corless et al. [9]). 
Proof. The quantile function is the root of the equation $\mathrm{F}_{X}\left(\mathrm{Q}_{X}(\mathrm{p})\right)=\mathrm{p}, \mathrm{p} \in(0,1)$. Therefore,

$$
\frac{c\left(\frac{\theta\left(\beta+\lambda+\beta \lambda\left(Q_{x}(p)\right)^{-\alpha}\right.}{\beta+\lambda} e^{\left.-\beta\left(Q_{x}(p)\right)^{-\alpha}\right)}\right.}{c(\theta)}=p .
$$

Multiplying both sides by $c(\theta)$ and applying $c^{-1}($.$) , then multiplying both sides by \frac{-(\beta+\lambda)}{\theta e^{\beta+\lambda}}$, leads to

$$
-\beta-\lambda-\beta \lambda\left(Q_{x}(p)\right)^{-\alpha} e^{-\beta-\lambda-\beta\left(Q_{x}(p)\right)^{-\alpha}}=\frac{-(\beta+\lambda) c^{-1}(p c(\theta))}{\theta e^{\beta+\lambda}} .
$$

Setting $z(p)=-\beta-\lambda-\beta \lambda\left(Q_{x}(p)\right)^{-\alpha}$, we have $z(p) e^{z(p)}=\frac{-(\beta+\lambda) c^{-1}(p c(\theta))}{\theta e^{\beta+\lambda}}$. The solution for $z(p)$ is $z(p)=W_{-1}\left[\frac{-(\beta+\lambda) c^{-1}(p c(\theta))}{\theta e^{\beta+\lambda}}\right]$, solving the equation $W_{-1}\left[\frac{-(\beta+\lambda) c^{-1}(p c(\theta))}{\theta e^{\beta+\lambda}}\right]=-\beta-\lambda-\beta \lambda\left(Q_{X}(p)\right)^{-\alpha}$ for $Q_{x}(p)$ completes the proof.

Order statistics are the most fundamental tools in non-parametric statistics and inference. These can be used to address estimation problems and hypothesis tests in many ways. The pdf of the $k^{\text {th }}$ order statistics from a random sample $X_{1}, \ldots, X_{n}$ from $X \sim \operatorname{GILPS}(\theta, \beta, \lambda, \alpha)$ is given by:

$$
\begin{aligned}
f_{k: n}(x) & =\frac{n !}{(k-1) !(n-k) !} f_{G I L P S}(x)\left[F_{G I L P S}(x)\right]^{k-1}\left[\mathbf{1}-F_{G I L P S}(x)\right]^{n-k} \\
& =\frac{n !}{(k-1) !(n-k) !} f_{X}(x) \sum_{i=0}^{n-k}\left(\begin{array}{c}
n-k \\
i
\end{array}\right)(-1)^{i}\left[F_{G I L P S}(x)\right]^{k+i-1} .
\end{aligned}
$$

The associated cdf can be defined as:

$$
F_{k: n}(x)=\frac{n !}{(k-1) !(k-i) !} \sum_{i=0}^{n-k} \frac{\left(\begin{array}{c}
n-k \\
i
\end{array}\right)(-1)^{i}}{k+i}\left[F_{G I L P S}(x)\right]^{k+i}
$$

where $F_{\text {GILPS }}(x)$ and $f_{\text {GILPS }}(x)$ come from (3.1) and (4.1), respectively.

\section{Estimation and inference}

Let $X_{1}, \ldots, X_{n}$ be a random sample with the observed value $x=\left(x_{1}, \ldots, x_{n}\right)$ obtained from the GILPS distribution with parameters $\theta, \beta, \lambda$, and $\alpha$. Let $\Theta=(\theta, \beta, \lambda, \alpha)^{\top}$ be the $4 \times 1$ parameter vector. The log likelihood function is given by:

$$
\begin{aligned}
l_{n}=l_{n}(\Theta ; x)= & n \log \alpha+n \log \theta+2 n \log \beta-n \log (\beta+\lambda)-n \log (c(\theta)) \\
& +\sum_{i=1}^{n} \log \left(x_{i}^{-\alpha-1}+\lambda x_{i}^{-2 \alpha-1}\right)-\beta \sum_{i=1}^{n} x_{i}^{-\alpha}+\sum_{i=1}^{n} \log \left[c^{\prime}\left(\frac{\theta\left(\beta+\lambda+\beta \lambda x^{-\alpha}\right)}{\beta+\lambda} e^{-\beta x^{-\alpha}}\right)\right] .
\end{aligned}
$$

Consider $p_{i}=\frac{\left(\beta+\lambda+\beta \lambda x^{-\alpha}\right)}{\beta+\lambda} e^{-\beta x^{-\alpha}}$. The score function, $u_{n}(\Theta)=\left(\partial l_{n} / \partial \theta, \partial l_{n} / \partial \beta, \partial l_{n} / \partial \lambda, \partial l_{n} / \partial \alpha\right)^{\top}$, is given by:

$$
\begin{array}{ll}
\frac{\partial l_{n}}{\partial \theta}=\frac{n}{\theta}-\frac{n c^{\prime}(\theta)}{c(\theta)}+\sum_{i=1}^{n} \frac{c^{\prime \prime}\left(\theta p_{i}\right) p_{i \theta}^{\prime}}{c^{\prime}\left(\theta p_{i}\right)}, & \frac{\partial l_{n}}{\partial \beta}=\frac{2 n}{\beta}-\frac{n}{\beta+\lambda}-\sum_{i=1}^{n} x_{i}^{-\alpha}+\sum_{i=1}^{n} \frac{c^{\prime \prime}\left(\theta p_{i}\right) p_{i \beta}^{\prime}}{c^{\prime}\left(\theta p_{i}\right)}, \\
\frac{\partial l_{n}}{\partial \lambda}=-\frac{n}{\beta+\lambda}+\sum_{i=1}^{n} \frac{1}{x_{i}^{\alpha}+\lambda}+\sum_{i=1}^{n} \frac{c^{\prime \prime}\left(\theta p_{i}\right) p_{i \lambda}^{\prime}}{c^{\prime}\left(\theta p_{i}\right)}, & \frac{\partial l_{n}}{\partial \alpha}=\frac{n}{\alpha}-\sum_{i=1}^{n} \frac{\left(1+2 \lambda x_{i}^{-\alpha}\right) \log x_{i}}{1+\lambda x_{i}^{-\alpha}}+\sum_{i=1}^{n} \frac{c^{\prime \prime}\left(\theta p_{i}\right) p_{i \alpha}^{\prime}}{c^{\prime}\left(\theta p_{i}\right)},
\end{array}
$$


where $p_{i \theta}^{\prime}=\frac{\partial p}{\partial \theta}, p_{i \beta}^{\prime}=\frac{\partial p}{\partial \beta}, p_{i \lambda}^{\prime}=\frac{\partial p}{\partial \lambda}$, and $p_{i \alpha}^{\prime}=\frac{\partial p}{\partial \alpha}$.

The maximum likelihood estimation (MLE) of $\Theta$, say $\widehat{\Theta}$, is obtained by solving the nonlinear system $\mathrm{U}_{n}(\mathrm{x} ; \Theta)=0$. Since this nonlinear system of equations does not have a closed form solution, any numerical method (such as the Newton-Raphson procedure) can be used. For the interval estimation and hypothesis tests on the model parameters, we require the following observed information matrix:

$$
\mathrm{I}_{n}(\Theta)=-\left[\begin{array}{cccc}
\mathrm{I}_{\theta \theta} & \mathrm{I}_{\theta \beta} & \mathrm{I}_{\theta \lambda} & \mathrm{I}_{\theta \alpha} \\
\mathrm{I}_{\beta \theta} & \mathrm{I}_{\beta \beta} & \mathrm{I}_{\beta \lambda} & \mathrm{I}_{\beta \alpha} \\
\mathrm{I}_{\lambda \theta} & \mathrm{I}_{\lambda \beta} & \mathrm{I}_{\lambda \lambda} & \mathrm{I}_{\lambda \alpha} \\
\mathrm{I}_{\alpha \theta} & \mathrm{I}_{\alpha \beta} & \mathrm{I}_{\alpha \lambda} & \mathrm{I}_{\alpha \alpha}
\end{array}\right],
$$

where the elements of $I_{n}(\Theta)$ are the second partial derivatives of $U_{n}(\Theta)$. Under the standard regularity conditions for the large sample approximation as mentioned in Cox and Hinkley [10], and verified here that these are fulfilled by the proposed model, the distribution of $\widehat{\Theta}$ is approximately $N_{p}\left(\Theta, J_{n}^{-1}(\Theta)\right.$ ), where $J_{n}(\Theta)=E\left[I_{n}(\Theta)\right]$. Whenever the parameters are in the interior of the parameter space and not on the boundary, the asymptotic distribution of $\sqrt{n}(\widehat{\Theta}-\Theta)$ is $N_{p}\left(0, J^{-1}(\Theta)\right)$, where $J^{-1}(\Theta)=\lim _{n \rightarrow \infty} n^{-1} I_{n}(\Theta)$ is the unit information matrix, and $p$ is the number of parameters of the distribution. The asymptotic multivariate normal $N_{p}\left(\Theta, I_{n}^{-1}(\widehat{\Theta})\right)$ distribution of $\widehat{\Theta}$ can be used to approximate the confidence interval for the parameters, the hazard rate and the survival functions. An $100(1-\gamma)$ asymptotic confidence interval for parameter $\Theta_{i}$ is given by

$$
\left(\widehat{\Theta}_{i}-Z_{\frac{\gamma}{2}} \sqrt{\widehat{I^{i i}}}, \widehat{\Theta}_{i}+Z_{\frac{\gamma}{2}} \sqrt{\widehat{I^{i i}}}\right),
$$

where $\widehat{I^{i i}}$ is the $(i, i)$ diagonal element of $I_{n}^{-1}(\widehat{\Theta})$ for $i=1, \ldots, p$, and $Z_{\frac{\gamma}{2}}$ is the quantile $1-\frac{\gamma}{2}$ of the standard normal distribution.

\section{Special cases of GILPS model}

In this section, we shall show that various models can be obtained as special cases of the GILPS class of distributions.

\subsection{Inverse power Lindley Poisson distribution}

The inverse power Lindley Poisson (IPLP) distribution (truncated at zero) is a special case of the GILPS distribution with $c(\theta)=e^{\theta}-1(\theta>0), \lambda=1$. Using (3.1), the cdf of the IPLP distribution is:

$$
F(x ; \theta, \beta, \alpha)=\frac{e^{\frac{\theta\left(\beta+1+\beta x^{-\alpha}\right)}{\beta+1}} e^{-\beta x^{-\alpha}}-1}{e^{\theta}-1}, \quad \theta, \beta, \alpha, x>0 .
$$

Using (4.1) and (4.2), the associated pdf and hf are given respectively by:

$$
\begin{aligned}
& f(x)=\frac{\alpha \theta \beta^{2}}{(\beta+1)\left(e^{\theta}-1\right)}\left(x^{-\alpha-1}+x^{-2 \alpha-1}\right) e^{-\beta x^{-\alpha}+\frac{\theta\left(\beta+1+\beta x^{-\alpha}\right)}{\beta+1}} e^{-\beta x^{-\alpha}}, \\
& \tau(x)=\frac{\alpha \theta \beta^{2}}{\beta+1} \frac{\left(x^{-\alpha-1}+x^{-2 \alpha-1}\right) e^{-\beta x^{-\alpha}+\frac{\theta\left(\beta+1+\beta x^{-\alpha}\right)}{\beta+1}} e^{-\beta x^{-\alpha}}}{e^{\theta}-e^{\frac{\theta\left(\beta+1+\beta x^{-\alpha}\right)}{\beta+1}} e^{-\beta x^{-\alpha}}}
\end{aligned}
$$

for $\theta, \beta, \alpha, x>0$.

Plots of the pdf and hf of the IPLP distribution for some selected parameter values are shown in Figure 1. 


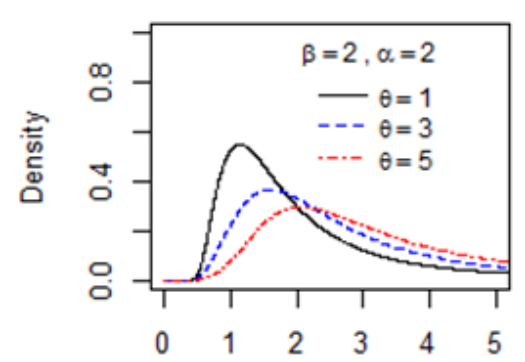

$\mathrm{x}$

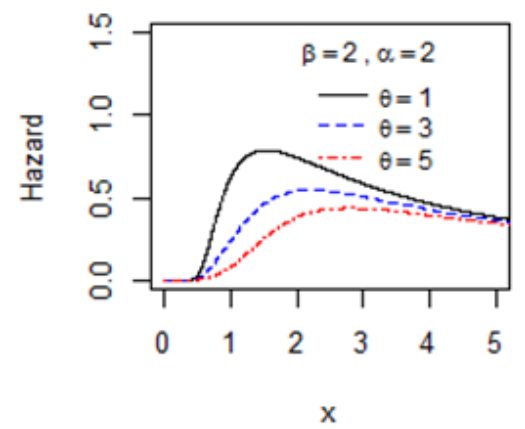

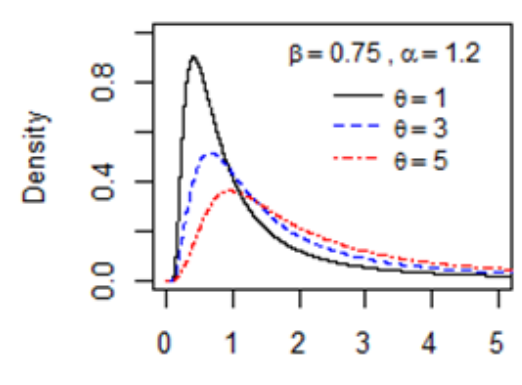

$\mathrm{x}$

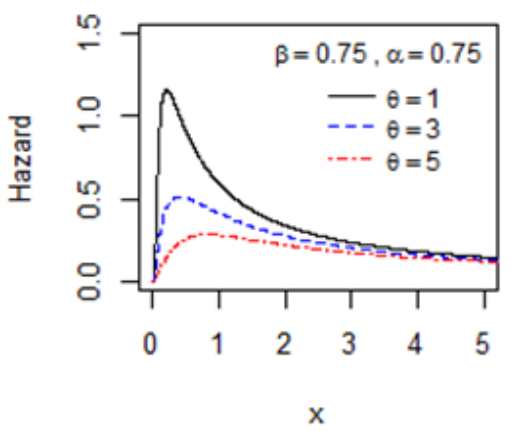

Figure 1: Plots of the density function of the IPLP distribution for different values of $\theta, \beta$, and $\alpha$.

The $r^{\text {th }}$ moment of a random variable $X$ following the IPLP distribution can be obtained from (4.4) with $a_{n}=\frac{1}{n !}$ :

$$
\mu_{r}^{\prime}=\sum_{n=1}^{\infty} \sum_{i=0}^{n-1}\left(\begin{array}{c}
n-1 \\
i
\end{array}\right) \frac{\theta^{n}}{e^{\theta}-1} n^{\frac{r}{\alpha}-n+i-1} \beta^{\frac{r}{\alpha}}(\beta+1)^{i-n}\left[n \beta+\left(n-\frac{r}{\alpha}-i\right)\right] \Gamma\left(n-\frac{r}{\alpha}-i\right) .
$$

From (4.5), the quantile function of the IPLP distribution can be readily expressed as

$$
Q_{X}(p)=\left[-1-\frac{1}{\beta}-\frac{1}{\beta} W_{-1}\left(\frac{-(\beta+1) \log \left(p\left(e^{\theta}-1\right)\right)}{\theta e^{\beta+1}}\right)\right]^{-\frac{1}{\alpha}} .
$$

For $\alpha=1$, we have the inverse Lindley Poisson (ILP) distribution.

\subsection{Inverse power Lindley geometric distribution}

The inverse power Lindley geometric (IPLG) distribution (truncated at zero) is a special case of the GILPS distribution with $c(\theta)=\frac{\theta}{1-\theta}, \lambda=1,(0<\theta<1)$. Using (3.1), the cdf of the IPLG distribution is:

$$
F(x ; \theta, \beta, \alpha)=\frac{(1-\theta)\left(\beta+1+\beta x^{-\alpha}\right)}{(\beta+1) e^{\beta x^{-\alpha}}-\theta\left(\beta+1+\beta x^{-\alpha}\right)} .
$$

From (4.1) and (4.2), the associated pdf and hf are given respectively by

$$
\begin{aligned}
& f(x)=\frac{\alpha \beta^{2}(\beta+1)(1-\theta)\left(x^{-\alpha-1}+x^{-2 \alpha-1}\right) e^{-\beta x^{-\alpha}}}{\left[\beta+1-\theta\left(\beta+1+\beta x^{-\alpha}\right) e^{\left.-\beta x^{-\alpha}\right]^{2}}\right.} \\
& \tau(x)=\frac{\alpha \beta^{2}(\beta+1)(1-\theta)\left(x^{-\alpha-1}+x^{-2 \alpha-1}\right)}{\left[(\beta+1) e^{\beta x^{-\alpha}}-\theta\left(\beta+1+\beta x^{-\alpha}\right)\right]\left[(\beta+1) e^{\beta x^{-\alpha}}-\left(\beta+1+\beta x^{-\alpha}\right)\right.}
\end{aligned}
$$


for $0<\theta<1, \beta, \alpha, x>0$.

Plots of the pdf and hf of the IPLG distribution for some selected parameter values are shown in Figure 2.

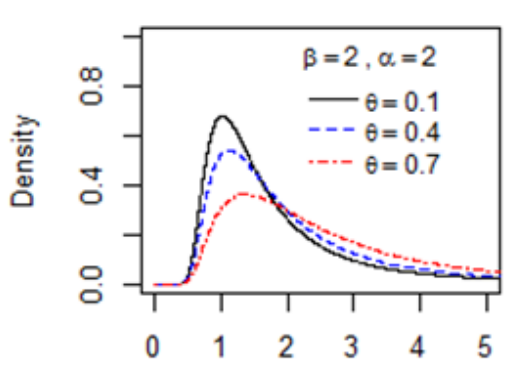

$\mathrm{X}$

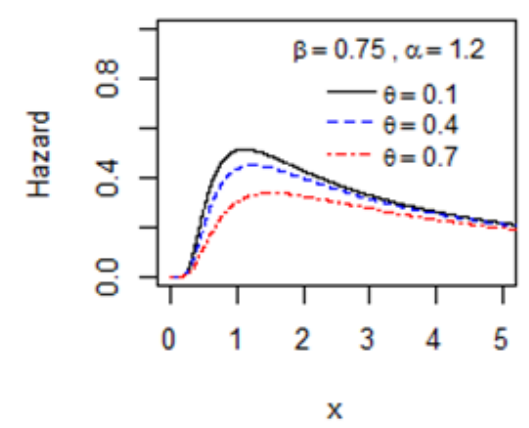

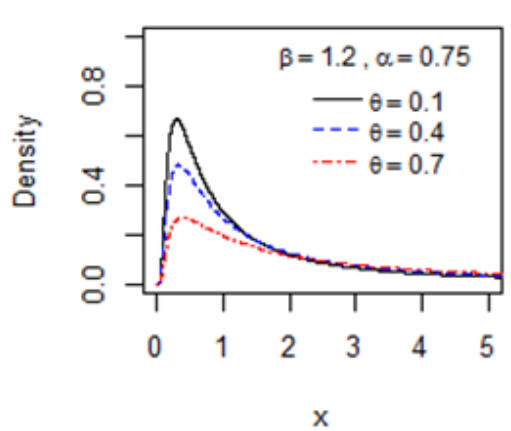

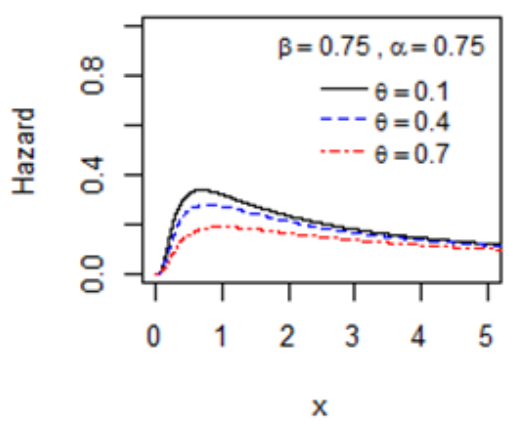

Figure 2: Plots of the density function of the IPLG distribution for different values of $\theta, \beta$, and $\alpha$.

The $r^{\text {th }}$ moment of a random variable $X$ following the IPLG distribution can be obtained from (4.4) with $a_{n}=1$ :

$$
\mu_{r}^{\prime}=\sum_{n=1}^{\infty} \sum_{i=0}^{n-1}\left(\begin{array}{c}
n-1 \\
i
\end{array}\right) \theta^{n-1}(1-\theta) \beta^{\frac{r}{\alpha}} n^{\frac{r}{\alpha}+i-n}(\beta+1)^{i-n}\left[n \beta+\left(n-\frac{r}{\alpha}-i\right)\right] \Gamma\left(n-\frac{r}{\alpha}-i\right) .
$$

From (4.5), the quantile function of the IPLG distribution can be readily defined by:

$$
\mathrm{Q}_{X}(\mathrm{p})=\left[-1-\frac{1}{\beta}-\frac{1}{\beta} \mathrm{W}_{-1}\left(\frac{-(\beta+1)}{\theta e^{\beta+1}} \frac{\mathrm{p} \theta}{\theta(\mathrm{p}-1)+1}\right)\right]^{-\frac{1}{\alpha}} .
$$

For $\alpha=1$, we have the inverse Lindley geometric (ILG) distribution.

\subsection{Inverse power Lindley logarithmic distribution}

The inverse power Lindley logarithmic (IPLL) distribution (truncated at zero) is a special case of the GILPS distribution with $c(\theta)=-\log (1-\theta), \lambda=1,(0<\theta<1)$. Using (3.1), the cdf of the IPLL distribution is:

$$
F(x ; \theta, \beta, \alpha)=\frac{1}{\log (1-\theta)} \log \left[1-\frac{\theta\left(\beta+1+\beta x^{-\alpha}\right)}{\beta+1} e^{-\beta x^{-\alpha}}\right] .
$$

By using (4.1) and (4.2) the associated pdf and hf are given respectively by:

$$
f(x)=\frac{-\theta \alpha \beta^{2}}{\log (1-\theta)} \frac{x^{-\alpha-1}+x^{-2 \alpha-1}}{(\beta+1) e^{\beta x^{-\alpha}}-\theta\left(\beta+1+\beta x^{-\alpha}\right)},
$$




$$
\tau(x)=\frac{-\theta \alpha \beta^{2}\left(x^{-\alpha-1}+x^{-2 \alpha-1}\right) e^{-\beta x^{-\alpha}}}{\left[(\beta+1)\left\{\log (1-\theta)-\log \left[1-\frac{\theta\left(\beta+1+\beta x^{-\alpha}\right)}{\beta+1} e^{-\beta x^{-\alpha}}\right]\right\}\left\{\beta+1-\left(\beta+1+\beta x^{-\alpha}\right) e^{-\beta x^{-\alpha}}\right\}\right.}
$$

for $0<\theta<1, \beta, \alpha, x>0$.

Plots of the pdf and hf of the IPLL distribution for some selected parameter values are shown in Figure 3.
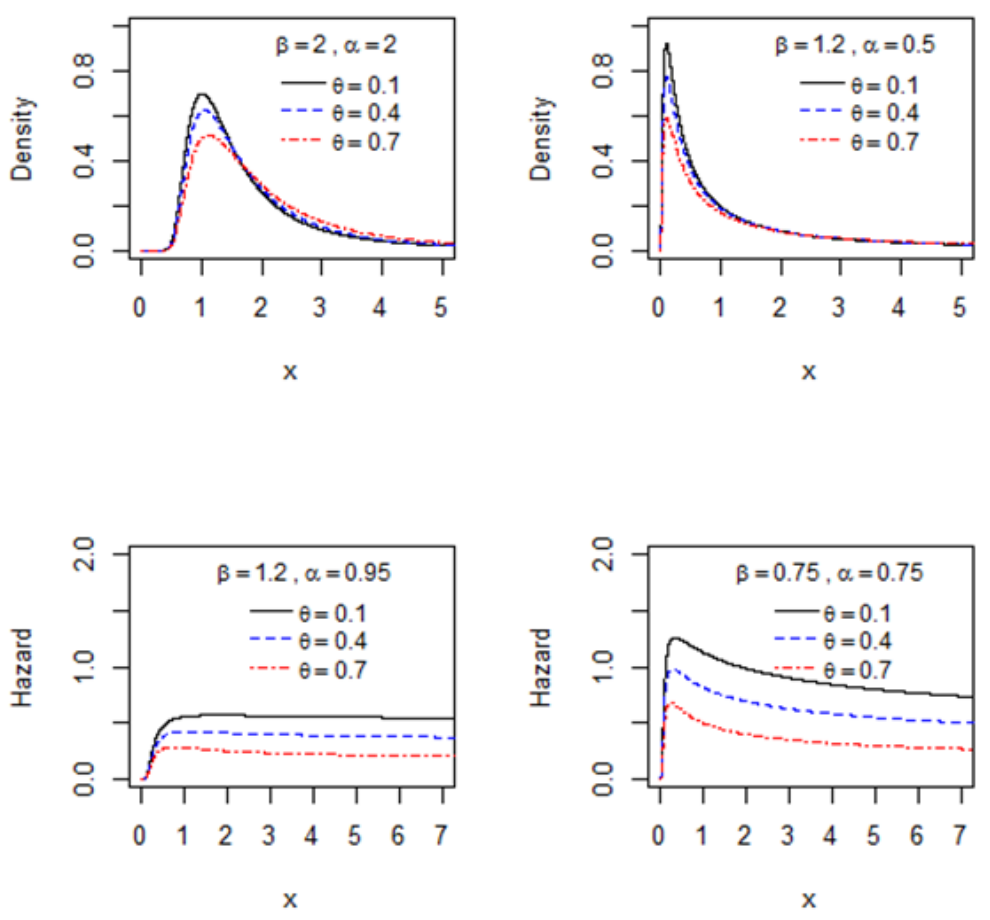

Figure 3: Plots of the density function of the IPLL distribution for different values of $\theta, \beta$, and $\alpha$.

The $r^{\text {th }}$ moment of a random variable $X$ following the IPLL distribution can be obtained from (4.4) with $a_{n}=\frac{1}{n}$ :

$$
\mu_{r}^{\prime}=\sum_{n=1}^{\infty} \sum_{i=0}^{n-1}\left(\begin{array}{c}
n-1 \\
i
\end{array}\right) \frac{\left(-\theta^{n}\right)}{\log (1-\theta)} \beta^{\frac{r}{\alpha}} n^{\frac{r}{\alpha}+i-n-1}(\beta+1)^{i-n}\left[n \beta+\left(n-\frac{r}{\alpha}-i\right)\right] \Gamma\left(n-\frac{r}{\alpha}-i\right) .
$$

From (4.5), the quantile function of the IPLL distribution can be expressed as:

$$
\mathrm{Q}_{x}(\mathrm{p})=\left[-1-\frac{1}{\beta}-\frac{1}{\beta} W_{-1}\left(\frac{-(\beta+1)\left(1-e^{p \log (1-\theta)}\right)}{\theta e^{\beta+1}}\right)\right]^{-\frac{1}{\alpha}} .
$$

For $\alpha=1$, we have the inverse Lindley logarithmic (ILL) distribution.

\subsection{Inverse power Lindley binomial distribution}

The inverse power Lindley binomial (IPLB) distribution (truncated at zero) is a special case of the GILPS distribution with $c(\theta)=(1+\theta)^{m}-1, \lambda=1,(0<\theta<1)$, where $m$ is a positive integer. Using (3.1), the cdf of the IPLB distribution is:

$$
F(x ; \theta, \beta, \alpha)=\frac{\left[\frac{\theta\left(\beta+1+\beta x^{-\alpha}\right)}{\beta+1} e^{-\beta x^{-\alpha}}+1\right]^{m}-1}{(\theta+1)^{m}-1} .
$$


By using (4.1) and (4.2), the associated pdf and hf are given respectively by:

$$
\begin{aligned}
f(x) & =\frac{\theta m \alpha \beta^{2}}{(\beta+1)^{m}} \frac{\left(x^{-\alpha-1}+x^{-2 \alpha-1}\right)\left[\theta\left(\beta+1+\beta x^{-\alpha}\right) e^{-\beta x^{-\alpha}}+\beta+1\right]^{m-1} e^{-\beta x^{-\alpha}}}{(\theta+1)^{m}-1}, \\
\tau(x) & =\frac{\theta m \alpha \beta^{2}\left(x^{-\alpha-1}+x^{-2 \alpha-1}\right)\left[\theta\left(\beta+1+\beta x^{-\alpha}\right) e^{-\beta x^{-\alpha}}+\beta+1\right]^{m-1} e^{-\beta x^{-\alpha}}}{(\beta+1)^{m}(\theta+1)^{m}-\left[\theta\left(\beta+1+\beta x^{-\alpha}\right) e^{-\beta x^{-\alpha}}+\beta+1\right]^{m}}
\end{aligned}
$$

for $0<\theta<1, \beta, \alpha, x>0$.

Plots of the pdf and hf of the IPLB distribution for some selected parameter values are shown in Figure 4.
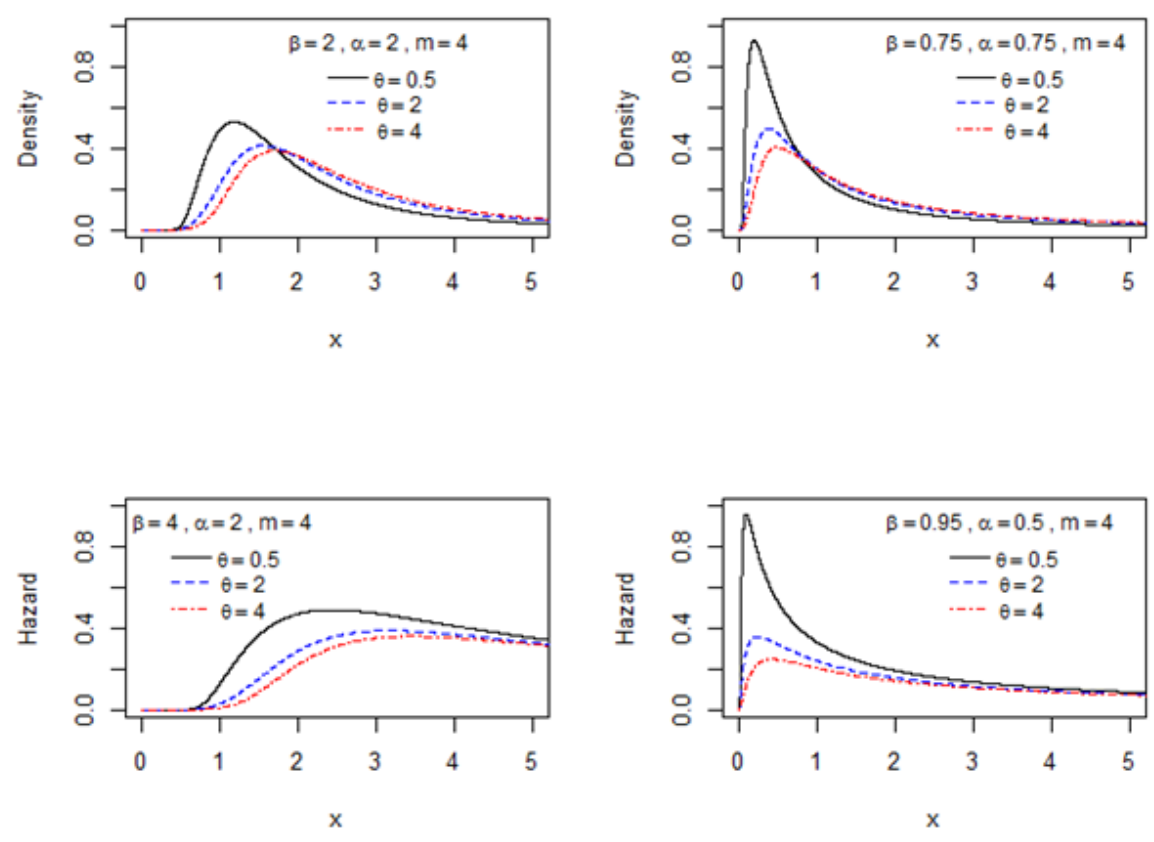

Figure 4: Plots of the density function of the IPLB distribution for different values of $\theta, \beta, \alpha$, and $m=4$.

The $r^{\text {th }}$ moment of a random variable $X$ following the IPLB distribution can be obtained from (4.4) with $a_{n}=\left(\begin{array}{c}m \\ n\end{array}\right)$ :

$$
\mu_{r}^{\prime}=\sum_{n=1}^{\infty} \sum_{i=0}^{n-1}\left(\begin{array}{c}
n-1 \\
i
\end{array}\right)\left(\begin{array}{c}
m \\
n
\end{array}\right) \frac{\theta^{n}}{(1+\theta)^{m}-1} \beta^{\frac{r}{\alpha}} n^{\frac{r}{\alpha}+i-n}(\beta+1)^{i-n}\left[n \beta+\left(n-\frac{r}{\alpha}-i\right)\right] \Gamma\left(n-\frac{r}{\alpha}-i\right) .
$$

From (4.5), the quantile function of the IPLB distribution can be expressed as:

$$
Q_{x}(p)=\left[-1-\frac{1}{\beta}-\frac{1}{\beta} W_{-1}\left(\frac{-(\beta+1)\left\{\left[p(1+\theta)^{m}-p+1\right]^{\frac{1}{m}}-1\right\}}{\theta e^{\beta+1}}\right)\right]^{-\frac{1}{\alpha}} .
$$

For $\alpha=1$, we have the inverse Lindley binomial (ILB) distribution.

\section{Simulation study}

In this section, we present the results of simulations for different sample sizes to assess the performances of the MLE estimators. For illustrative purposes, we choose the IPLG distribution. The simulation technique for the IPLG distribution is the inversion method using the following lemma. 
Lemma 7.1. Let $\mathrm{U}$ be a standard uniform variable between zero and one. Then, the random variable:

$$
X=\left[-1-\frac{1}{\beta}-\frac{1}{\beta} W_{-1}\left(\frac{-(\beta+1)}{\theta e^{\beta+1}} \frac{\mathrm{u} \theta}{\theta(\mathrm{U}-1)+1}\right)\right]^{-\frac{1}{\alpha}},
$$

is said to come from the IPLG distribution with parameters $\theta, \beta$, and $\alpha$.

Samples of size $n=25,50,100,200,400$, and 600 are generated for two sets of parameters, namely $(\theta=0.2, \beta=0.8, \alpha=0.75)$ and $(\theta=0.8, \beta=1.1, \alpha=2)$. Using Lemma 7.1, we repeated the simulations for $N=5000$, and evaluated the average estimate (AE), average bias (AB), and root mean squared error (RMSE). The empirical results are obtained by using the statistical computing software $\mathrm{R}$ and are presented in Table 2. It can be seen that as the sample size increases, the RMSE and AB decrease toward zero and the AE converges to the actual parameter. Thus, we can conclude that the MLE method provides consistent estimates of the model parameters.

Table 2: The AE, AB, and RMSE for varying $n, \theta, \beta$, and $\alpha$.

\begin{tabular}{|c|c|c|c|c|c|c|c|}
\hline \multicolumn{3}{|c|}{$\theta=0.2, \beta=0.8, \alpha=0.75$} & \multicolumn{3}{c|}{$\theta=0.8, \beta=1.1, \alpha=2$} \\
\hline Par. & $\mathrm{n}$ & $\mathrm{AE}$ & $\mathrm{AB}$ & $\mathrm{RMSE}$ & $\mathrm{AE}$ & $\mathrm{AB}$ & $\mathrm{RMSE}$ \\
\hline \multirow{5}{*}{$\theta$} & 25 & 0.3205 & 0.1205 & 0.3597 & 0.4801 & -0.3199 & 0.4686 \\
\cline { 2 - 8 } & 50 & 0.3115 & 0.1115 & 0.3441 & 0.5494 & -0.2506 & 0.4029 \\
\cline { 2 - 8 } & 100 & 0.3057 & 0.1057 & 0.3285 & 0.6352 & -0.1648 & 0.3123 \\
\cline { 2 - 8 } & 200 & 0.2934 & 0.0934 & 0.3005 & 0.7073 & -0.0927 & 0.2128 \\
\cline { 2 - 8 } & 400 & 0.2545 & 0.0545 & 0.2469 & 0.7576 & -0.0424 & 0.1319 \\
\cline { 2 - 8 } & 600 & 0.2405 & 0.0405 & 0.2249 & 0.7743 & -0.0257 & 0.0984 \\
\hline \multirow{5}{*}{$\beta$} & 25 & 0.6968 & -0.1032 & 0.2805 & 1.7280 & 0.6280 & 0.9525 \\
\cline { 2 - 8 } & 50 & 0.7064 & -0.0936 & 0.2507 & 1.5581 & 0.4581 & 0.7408 \\
\cline { 2 - 8 } & 100 & 0.7155 & -0.0845 & 0.2298 & 1.3941 & 0.2941 & 0.5632 \\
\cline { 2 - 8 } & 200 & 0.7257 & -0.0743 & 0.2006 & 1.2680 & 0.1680 & 0.4080 \\
\cline { 2 - 8 } & 400 & 0.7572 & -0.0428 & 0.1540 & 1.1759 & 0.0759 & 0.2886 \\
\cline { 2 - 8 } & 600 & 0.7668 & -0.0332 & 0.1374 & 1.1456 & 0.0456 & 0.2331 \\
\hline \multirow{5}{*}{$\alpha$} & 25 & 0.8346 & 0.0846 & 0.1748 & 1.9462 & -0.0538 & 0.3890 \\
\cline { 2 - 8 } & 50 & 0.8056 & 0.0556 & 0.1247 & 1.9158 & -0.0842 & 0.2993 \\
\cline { 2 - 8 } & 100 & 0.7909 & 0.0409 & 0.0991 & 1.9282 & -0.0718 & 0.2388 \\
\cline { 2 - 7 } & 200 & 0.7822 & 0.0322 & 0.0781 & 1.9594 & -0.0406 & 0.1809 \\
\cline { 2 - 7 } & 400 & 0.680 & 0.0180 & 0.0554 & 1.9828 & -0.0172 & 0.1334 \\
\cline { 2 - 8 } & 600 & 0.7633 & 0.0133 & 0.0485 & 1.9914 & -0.0086 & 0.1083 \\
\hline
\end{tabular}

\section{Applications}

In this section, we fit the IPLP, IPLG, IPLL, and IPLB to three real datasets and compare the results with two three-parameter distributions. The first is the generalized inverse Weibull (GIW) distribution introduced by Gusmão et al. [11]. The cdf of the GIW distribution is given by

$$
F(x ; \theta, \beta, \alpha)=e^{-\theta\left(\frac{\alpha}{x}\right)^{\beta}}, \theta, \beta, \alpha, x>0 .
$$

The second is the extended inverse Lindley (EIL) distribution proposed by Alkarni [1]. The cdf of the EIL is given by

$$
F(x ; \theta, \beta, \alpha)=\left[1+\frac{\theta \beta}{\theta+\beta} \frac{1}{x^{\alpha}}\right] e^{-\frac{\theta}{x^{\alpha}}}, \quad \theta, \beta, \alpha, x>0 .
$$


The first data set represents the relief times (in minutes) of 20 patients receiving an analgesic (reported by Gross and Clark [15]). These data consist of 20 observations and have the following values: 1.1, 1.4, $1.3,1.7,1.9,1.8,1.6,2.2,1.7,2.7,4.1,1.8,1.5,1.2,1.4,3.0,1.7,2.3,1.6$, and 2.0.

The second data corresponds to 46 observations reported on active repair times (hours) for an airborne communication transceiver discussed by Alven [3]. The data are: $0.2,0.3,0.5,0.5,0.5,0.5,0.6,0.6,0.7,0.7$, $0.7,0.8,0.8,1.0,1.0,1.0,1.0,1.1,1.3,1.5,1.5,1.5,1.5,2.0,2.0,2.2,2.5,2.7,3.0,3.0,3.3,3.3,4.0,4.0,4.5,4.7$, $5.0,5.4,5.4,7.0,7.5,8.8,9.0,10.3,22.0,24.5$.

The third data set represents the flood levels for the Susquehanna River at Harrisburg, Pennsylvania, over 20 four-year periods from 1890 to 1969 and was obtained in a civil engineering context and give the maximum flood level (in millions of cubic feet per second). This data has been widely used by authors and were initially reported by Dumonceaux and Antle [12]. The values of this data are: $0.654,0.613,0.315$, $0.449,0.297,0.402,0.379,0.423,0.379,0.324,0.269,0.740,0.418,0.412,0.494,0.416,0.338,0.392,0.484,0.265$.

For each distribution, we derive the maximum likelihood estimates (MLE), the maximized log likelihood (Log L), the Kolmogorov-Smirnov statistics (K-S) with their respective p-value, the Akaike Information Criterion (AIC), and the Bayesian Information Criterion (BIC). The K-S test is valid to test the quality of the fit of the underlying distributions to the failure data, as shown in Bagheri et al. [4]. The results of all data sets are presented in Table 3,4, and 5. The fitted densities of GILPS models and the competitor's models for all the data sets are shown in Figures 5, 6, and 7. These indicate that the GILPS distributions fits the data better than the other distributions. The KS test statistic has smallest value with largest p-value for the GILPS distributions. This is confirmed by the log likelihood, the AIC and the BIC also.

Table 3: Parameter estimates, KS statistic, P-value, log likelihood, AIC, and BIC of relief times data.

\begin{tabular}{|c|c|c|c|c|c|c|c|c|}
\hline Dist. & $\hat{\theta}$ & $\hat{\beta}$ & $\hat{\alpha}$ & K-S & p-value & $-\log (\mathrm{L})$ & $\mathrm{AIC}$ & $\mathrm{BIC}$ \\
\hline IPLP & 1.2114 & 5.7405 & 4.4234 & 0.0880 & 0.9939 & 15.38 & 36.76 & 39.74 \\
\hline IPLG & 0.3820 & 6.0742 & 4.3965 & 0.0877 & 0.9941 & 15.39 & 36.76 & 39.74 \\
\hline IPLL & 0.4759 & 6.3464 & 4.2787 & 0.0853 & 0.9959 & 15.40 & 36.80 & 39.78 \\
\hline IPLB & 0.1444 & 5.7078 & 4.4048 & 0.0790 & 0.9986 & 15.36 & 36.75 & 39.74 \\
\hline EIL & 6.0286 & 0.0062 & 4.0175 & 0.0886 & 0.9934 & 15.41 & 36.82 & 39.80 \\
\hline GIW & 1.1396 & 4.0175 & 1.5135 & 0.0897 & 0.9923 & 15.41 & 36.82 & 39.80 \\
\hline
\end{tabular}

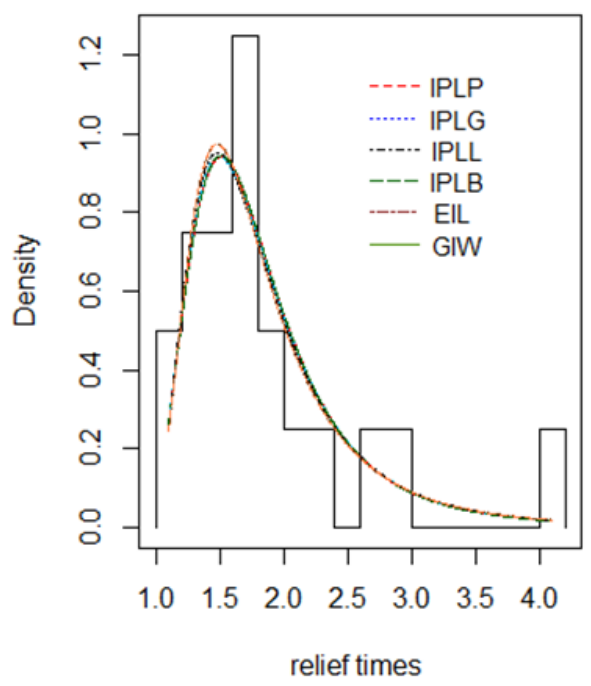

Figure 5: Plots of fitted models of the relief times data. 
Table 4: Parameter estimates, KS statistic, P-value, log likelihood, AIC, and BIC of active repair times data.

\begin{tabular}{|c|c|c|c|c|c|c|c|c|}
\hline Dist. & $\hat{\theta}$ & $\hat{\beta}$ & $\hat{\alpha}$ & K-S & p-value & $-\log (\mathrm{L})$ & AIC & BIC \\
\hline IPLP & 2.5689 & 0.8989 & 1.0700 & 0.0795 & 0.9106 & 100.45 & 206.89 & 212.38 \\
\hline IPLG & 0.8783 & 0.5691 & 1.2594 & 0.0717 & 0.9584 & 100.07 & 206.14 & 211.62 \\
\hline IPLL & 0.9947 & 0.4503 & 1.6347 & 0.0759 & 0.9357 & 99.83 & 205.67 & 211.15 \\
\hline IPLB & 0.3032 & 0.9454 & 1.0530 & 0.0796 & 0.9104 & 100.49 & 206.98 & 212.46 \\
\hline EIL & 1.1316 & 0.00001 & 1.0127 & 0.0798 & 0.9085 & 100.69 & 207.38 & 212.87 \\
\hline GIW & 0.4788 & 1.0127 & 2.3380 & 0.0803 & 0.905 & 100.69 & 207.38 & 212.87 \\
\hline
\end{tabular}

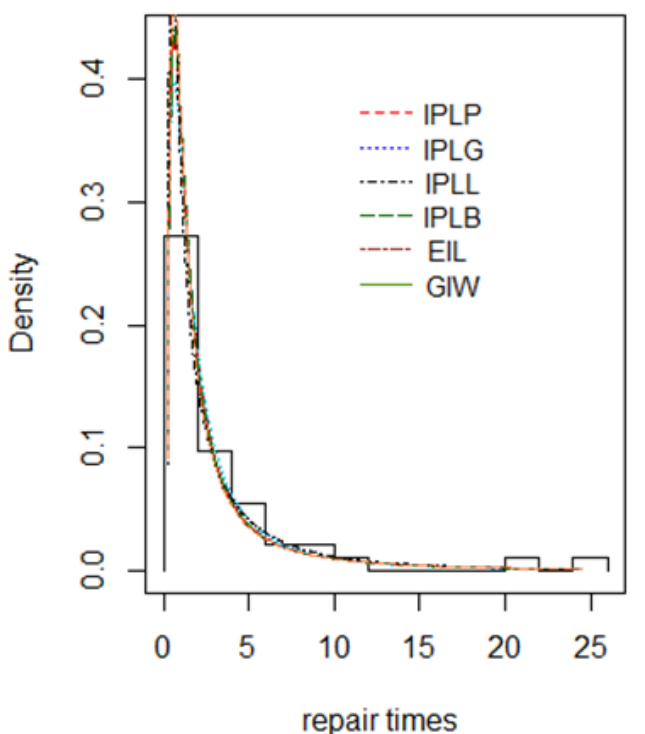

Figure 6: Plots of fitted models of the repair times data.

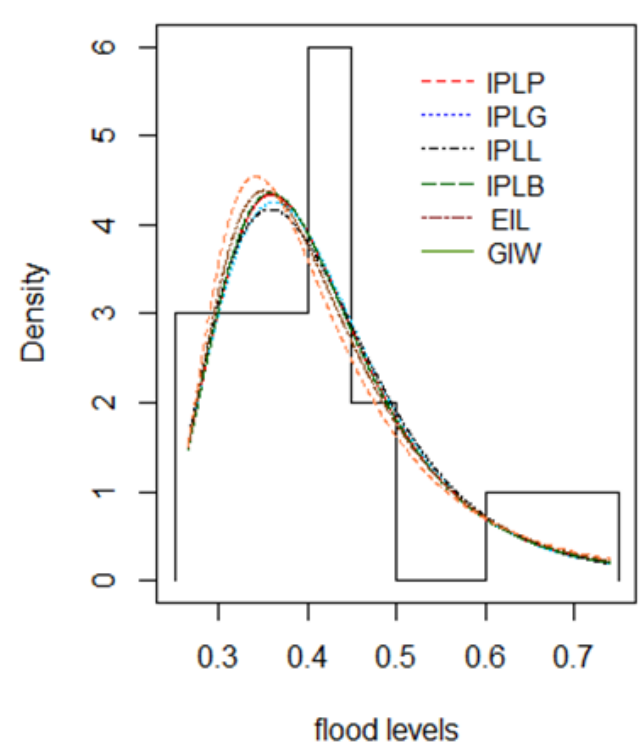

Figure 7: Plots of fitted models of the flood levels data.

Table 5: Parameter estimates, KS statistics, P-value, log likelihood, AIC, and BIC the flood levels data
\begin{tabular}{|c|c|c|c|c|c|c|c|c|}
\hline Dist. & $\hat{\theta}$ & $\hat{\beta}$ & $\hat{\alpha}$ & K-S & p-value & $-\log (\mathrm{L})$ & AIC & BIC \\
\hline IPLP & 1.1918 & 0.0583 & 3.2719 & 0.1295 & 0.8491 & -16.23 & -26.47 & -23.48 \\
\hline IPLG & 0.5622 & 0.0460 & 3.4086 & 0.1250 & 0.8757 & -16.26 & -26.52 & -23.54 \\
\hline IPLL & 0.8379 & 0.0390 & 3.5432 & 0.1274 & 0.8615 & -16.27 & -26.53 & -23.55 \\
\hline IPLB & 0.1324 & 0.0602 & 3.2555 & 0.1302 & 0.8444 & -16.23 & -26.46 & -23.47 \\
\hline EIL & 0.1056 & 4.2781 & 2.9546 & 0.1388 & 0.7865 & -16.23 & -26.47 & -23.48 \\
\hline GIW & 0.1214 & 4.3143 & 0.5842 & 0.1546 & 0.6698 & -16.10 & -26.19 & -23.28 \\
\hline
\end{tabular}

\section{Concluding remarks}

The purpose of this paper was to define a new family of lifetime distributions called the GILPS family of distributions. The GILPS class contains some lifetime subclasses and has the ability to produce many useful and flexible distributions for modeling lifetime data. The properties of the GILPS class of distributions have been derived in flexible and useful forms, including density, hazard function, quantile function, moments, moments generating function, distribution of order statistics and maximum likelihood estimates. Some subclasses and models were introduced to show the beneficiary of the proposed class. A simulation was conducted to test the estimating method performance for the model parameters. In 
addition, some of the GILPS distributions applied to three real data sets and then compared to some existing distributions.

\section{Acknowledgment}

The author is grateful to the Deanship of Scientific Research at King Saud University, as represented by the Research Center of the College of Business Administration, for financially supporting this study.

\section{References}

[1] S. H. Alkarni, Extended inverse Lindley distribution: properties and application, Springer-Plus, 4 (2015), 1-13. 1, 2.1, 8

[2] S. H. Alkarni, Generalized extended Weibull power series family of distributions, J. Data Sci., 14 (2016), 415-440. 1

[3] W. Alven, Reliability Engineering by ARINC, Prentice-hall, New Jersey, (1964). 8

[4] S. F. Bagheri, E. B. Bahrani, M. Ganjali, The generalized modified Weibull power series distribution: Theory and applications, Comput. Statist. Data Anal., 94 (2016), 136-160. 1, 8

[5] K. V. P. Barco, J. Mazucheli, V. Janeiro, The inverse power Lindley distribution, Comm. Statist. Simulation Comput., 46 (2017), 6308-6323. 1, 2.1.3

[6] H. Bidram, V. Nekoukhou, Double bounded Kumaraswamy-power series class of distributions, SORT, 37 (2013), $211-229$. 1

[7] M. Bourguignon, R. B. Silva, G. M. Cordeiro, A new class of fatigue life distributions, J. Stat. Comput. Simul., 84 (2014), 2619-2635. 1

[8] G. M. Cordeiro, R. B. Silva, The complementary extended Weibull power series class of distributions, Ciência e Natura, 36 (2014), 1-13. 1

[9] R. M. Corless, G. H. Gonnet, D. E. G. Hare, D. J. Jeffrey, D. E. Knuth, On the Lambert W function, Adv. Comput. Math., 5 (1996), 329-359. 4.3

[10] D. R. Cox, D. V. Hinkley, Theoretical Statistics, Chapman and Hall, London, (1974). 5

[11] F. R. S. de Gusmão, E. M. M. Ortega, G. M. Cordeiro, The generalized inverse Weibull distribution, Statist. Papers, 52 (2011), 591-619. 8

[12] R. Dumonceaux, C. E. Antle, Discrimination between the Log-Normal and the Weibull Distributions, Technometrics, 15 (1973), 923-926. 8

[13] I. Elbatal, M. Zayed, M. Rasekhi, N. S. Butt, The Exponential Pareto Power Series Distribution: Theory and Applications, Pak. J. Stat. Oper. Res., 13 (2017), 603-615. 1

[14] J. Flores, P. Borges, V. G. Cancho, F. Louzada, The complementary exponential power series distribution, Braz. J. Probab. Stat., 27 (2013), 565-584. 1

[15] A. J. Gross, V. Clark, Survival Distributions: Reliability Applications in the Biometrical Sciences, John Wiley \& Sons, New York, (1975). 8

[16] S. S. Harandi, M. H. Alamatsaz, Generalized linear failure rate power series distribution, Comm. Statist. Theory Methods, 45 (2016), 2204-2227. 1

[17] A. S. Hassan, A. M. Abd-Elfattah, A. H. Mokhtar, The Complementary Exponentiated Inverted Weibull Power Series Family of Distributions and its Applications, British J. Math. Comput. Sci., 13 (2016), 1-20. 1

[18] A. S. Hassan, M. S. Assar, K. A. Ali, The Compound Family of Generalized Inverse Weibull Power Series Distributions, British J. Appl. Sci. Tech., 14 (2016), 1-18. 1

[19] A. A. Jafari, S. Tahmasebi, Gompertz-power series distributions, Comm. Statist. Theory Methods, 45 (2016), 37613781. 1

[20] N. L. Johnson, A. W. Kemp, S. Kotz, Univariate Discrete Distributions: third edition, John Wiley \& Sons, Hoboken, (2005). 3

[21] D. V. Lindley, Fiducial distributions and Bayes' theorem, J. Roy. Statist. Soc. Ser. B, 20 (1958), 102-107. 1

[22] W. Liyanage, G. J. D. A. Gunathilake, The Lindley power series class of distributions: model, properties and Applications, J. Comput. Model., 5 (2015), 35-80. 1

[23] E. Mahmoudi, A. A. Jafari, Generalized exponential-power series distributions, Comput. Statist. Data Anal., 56 (2012), 4047-4066. 1

[24] E. Mahmoudi, A. A. Jafari, The compound class of linear failure rate-power series distributions: model, properties and applications, arXiv preprint, 2014 (2014), 39 pages. 1

[25] A. L. Morais, W. Barreto-Souza, A compound class of Weibull and power series distributions, Comput. Statist. Data Anal., 55 (2011), 1410-1425. 1

[26] S. Nadarajah, B. V. Popović, M. M. Ristić, Compounding: An R package for computing continuous distributions obtained by compounding a continuous and a discrete distribution, Comput. Statist., 28 (2013), 977-992. 1

[27] S. Shafie, S. Darijani, H. Saboori, Inverse Weibull power series distributions: properties and applications, J. Stat. Comput. Simul., 86 (2016), 1069-1094. 1

[28] V. K. Sharma, P. Khandelwal, On the extension of inverse Lindley distribution, J. Data Sci., 15 (2017), 205-220. 1 
[29] V. K. Sharma, S. K. Singh, U. Singh, V. Agiwal, The inverse Lindley distribution: A stress-strength reliability model with applications to head and neck cancer data, J. Ind. Prod. Eng., 32 (2015), 162-173. 1, 2.1 .1

[30] V. K. Sharma, S. K. Singh, U. Singh, F. Merovci, The generalized inverse Lindley distribution: A new inverse statistical model for the study of upside-down bathtub survival data, Comm. Statist. Theory Methods, 45 (2016), 5709-5729. 1

[31] R. B. Silva, M. Bourguignon, C. R. B. Dias, G. M. Cordeiro, The compound class of extended Weibull power series distributions, Comput. Stat. Data Anal., 58 (2013), 352-367. 1

[32] R. B. Silva, G. M. Cordeiro, The Burr XII power series distributions: A new compounding family, Braz. J. Probab. Stat., 29 (2015), 565-589. 1

[33] S. Tahmasebi, A. A. Jafari, Exponentiated extended Weibull-power series class of distributions, Ciência e Natura, 37 (2015), 183-193. 1

[34] R. C. Team, R: A Language and Environment for Statistical Computing, R Foundation for Statistical Computing, Vienna, (2014). 1 